\title{
Genetically Dynamic Optimization Based Fuzzy Polynomial Neural Networks
}

\author{
Ho-Sung Park ${ }^{1}$, Sung-Kwun $\mathrm{Oh}^{2}$, Witold Pedrycz ${ }^{3}$, and Yongkab Kim ${ }^{1}$ \\ ${ }^{1}$ Department of Electrical Electronic and Information Engineering, Wonkwang University, \\ 344-2, Shinyong-Dong, Iksan, Chon-Buk, 570-749, South Korea \\ ${ }^{2}$ Department of Electrical Engineering, The University of Suwon, San 2-2 Wau-ri, \\ Bongdam-eup, Hwaseong-si, Gyeonggi-do, 445-743, South Korea \\ ohsk@suwon.ac.kr \\ ${ }^{3}$ Department of Electrical and Computer Engineering, University of Alberta, \\ Edmonton, AB T6G 2G6, Canada \\ and Systems Research Institute, Polish Academy of Sciences, Warsaw, Poland
}

\begin{abstract}
In this paper, we introduce a new architecture of genetically dynamic optimization based Fuzzy Polynomial Neural Networks (gdFPNN) and discuss its comprehensive design methodology involving mechanisms of genetic optimization, especially genetic algorithms (GAs). The proposed gdFPNN gives rise to a structurally and parametrically optimized network through an optimal parameters design available within FPN. Through the consecutive process of such structural and parametric optimization, an optimized and flexible gdFPNN is generated in a dynamic fashion. The performance of the proposed gdFPNN is quantified through experimentation that exploits standard data already used in fuzzy modeling. These results reveal superiority of the proposed networks over the existing fuzzy and neural models.
\end{abstract}

\section{Introduction}

The challenging quest for constructing models of the systems that come with significant approximation and generalization abilities as well as are easy to comprehend has been within the community for decades [1], [2], [3], [4]. The most successful approaches to hybridize fuzz systems with learning and adaptation have been made in the realm of CI [5]. As one of the representative design approaches which are advanced tools, a family of fuzzy polynomial neuron (FPN)-based SOPNN(called "FPNN" as a new category of neuro-fuzzy networks)[6] were introduced to build predictive models for such highly nonlinear systems. The FPNN algorithm exhibits some tendency to produce overly complex networks as well as a repetitive computation load by the trial and error method and/or the repetitive parameter adjustment by designer like in case of the original GMDH algorithm.

In this study, in addressing the above problems with the conventional SOPNN (especially, FPN-based SOPNN called "FPNN" [6], [7]) as well as the GMDH algorithm, we introduce a new genetic design approach; as a consequence we will be referring to these networks as genetically dynamic optimization based FPNN (to be 
called "gdFPNN"). The determination of the optimal values of the parameters available within an individual FPN (viz. the number of input variables, the order of the polynomial, input variables, the number of membership function, and the apexes of membership function) leads to a structurally and parametrically optimized network.

\section{The Architecture and Development of Fuzzy Polynomial Neural Networks (FPNN)}

\subsection{FPNN Based on Fuzzy Polynomial Neurons (FPNs)}

The FPN consists of two basic functional modules. The first one, labeled by $\mathbf{F}$, is a collection of fuzzy sets that form an interface between the input numeric variables and the processing part realized by the neuron. The second module (denoted here by $\mathbf{P}$ ) is about the function - based nonlinear (polynomial) processing. This nonlinear processing involves some input variables.

\subsection{The Review of Conventional FPNN Architecture}

Proceeding with the conventional FPNN architecture as presented in [6], [7], essential design decisions have to be made with regard to the number of input variables and the order of the polynomial occurring in the conclusion part of the rule. The overall selection process of the conventional FPNN architecture is shown in Fig. 1

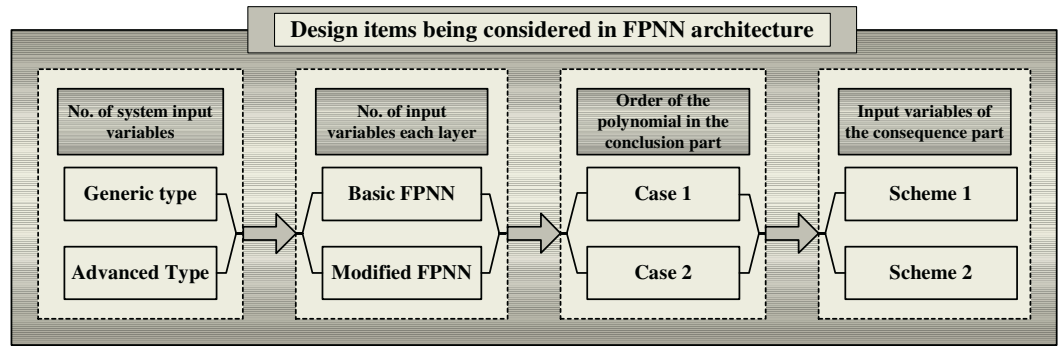

Fig. 1. Taxonomy of the conventional FPNN architecture

\section{The Algorithms and Design Procedure of Genetically Dynamic Optimization Based FPNN}

\subsection{Genetic Optimization of FPNN}

GAs is optimization techniques based on the principles of natural evolution. In essence, they are search algorithms that use operations found in natural genetics to guide a comprehensive search over the parameter space. GAs has been theoretically and empirically demonstrated to provide robust search capabilities in complex spaces thus offering a valid solution strategy to problems requiring efficient and effective searching [8]. In this study, for the optimization of the FPNN model, GA uses the 
serial method of binary type, roulette-wheel used in the selection process, one-point crossover in the crossover operation, and a binary inversion (complementation) operation in the mutation operator. To retain the best individual and carry it over to the nest generation, we use elitist strategy [9].

\subsection{Design Procedure of Genetically Dynamic Optimization Based FPNN}

[Step 1] Determine system's input variables

[Step 2] Form training and testing data

The input-output data set $\left(\boldsymbol{x}_{i}, y_{i}\right)=\left(x_{1 i}, x_{2 i}, \ldots, x_{n i}, y_{i}\right), i=1,2, \ldots, N$ is divided into two parts, that is, a training and testing dataset.

[Step 3] Decide initial information for constructing the gdFPNN structure [Step 4] Decide FPN structure using genetic design

We divide the chromosome to be used for genetic optimization into four subchromosomes. The $1^{\text {st }}$ sub-chromosome contains the number of input variables, the $2^{\text {nd }}$ sub-chromosome involves the order of the polynomial of the node, the $3^{\text {rd }}$ subchromosome contains input variables, and the $4^{\text {th }}$ sub-chromosome (remaining bits) involves the number of MF coming to the corresponding node (FPN).

[Step 5] Design of structurally optimized gdFPNN

In this step, we design the structurally optimized gdFPNN by means of FPNs that obtained in [Step 4].

[Step 6] Identification of membership value using dynamic searching method of GAs [Step 7] Design of parametrically optimized gdFPNN

Sub-step 1) We set up initial genetic information necessary for generation of the gdFPNN architecture.

Sub-step 2) The nodes (FPNs) are generated through the genetic design.

Sub-step 4) we calculate the fitness function.

Sub-step 5) To move on to the next generation, we carry out selection, crossover, and mutation operation using genetic initial information and the fitness values.

Sub-step 6) We choose optimal gdFPNN characterized by the best fitness value in the current generation. For the elitist strategy, selected best fitness value used.

Sub-step 7) We generate new populations of the next generation using operators of GAs obtained from Sub-step 2. We use the elitist strategy. This sub-step carries out by repeating sub-step 2-6.

Sub-step 8) Until the last generation, this sub-step carries out by repeating sub-step 2-7.

\section{Experimental Studies}

The performance of the gdFPNN is illustrated with the aid of well-known and widely used dataset of a gas furnace process utilized by Box and Jenkins [10].

We try to model the gas furnace using 296 pairs of input-output data. The total data set consisting 296 input-output pairs was split into two parts. The first one (consisting of 148 pairs) was used for training. The remaining part of the series serves as a testing 
set. In order to carry out the simulation, we use six-input $[u(t-3), u(t-2), u(t-1), y(t-3)$, $y(t-2), y(t-1)]$ and one-output $(y(t))$. To come up with a quantitative evaluation of the network, we use the standard MSE performance index.

Table 1 summarizes the performance index of gdFPNN when using dynamic searching method.

Table 1. Performance index of gdFPNN for the gas furnace process data (3rd layer)

\begin{tabular}{ccccccccc}
\hline & \multicolumn{3}{c}{ (a) Selected input variables } & \multicolumn{3}{c}{ (b) Entire system input variables } \\
\hline \multirow{2}{*}{ Max } & \multicolumn{2}{c}{ Triangular MF } & \multicolumn{2}{c}{ Gaussian-like MF } & \multicolumn{2}{c}{ Triangular MF } & \multicolumn{2}{c}{ Gaussian-like MF } \\
& PI & EPI & PI & EPI & PI & EPI & PI & EPI \\
2 & 0.019 & 0.102 & 0.013 & 0.101 & 0.011 & 0.105 & 0.012 & 0.102 \\
3 & 0.015 & 0.115 & 0.011 & 0.116 & 0.011 & 0.110 & 0.007 & 0.106 \\
\hline
\end{tabular}

Fig. 2 illustrates the detailed optimal topologies of gdFPNN for 3 layer when using $\mathrm{Max}=2$ and Gaussian-like MF: the results of the network have been reported as $\mathrm{PI}=0.012$ and $\mathrm{EPI}=0.102$.

As shown in Fig 2, the proposed network enables the architecture to be a structurally and parametrically more optimized and simplified network than the conventional FPNN.

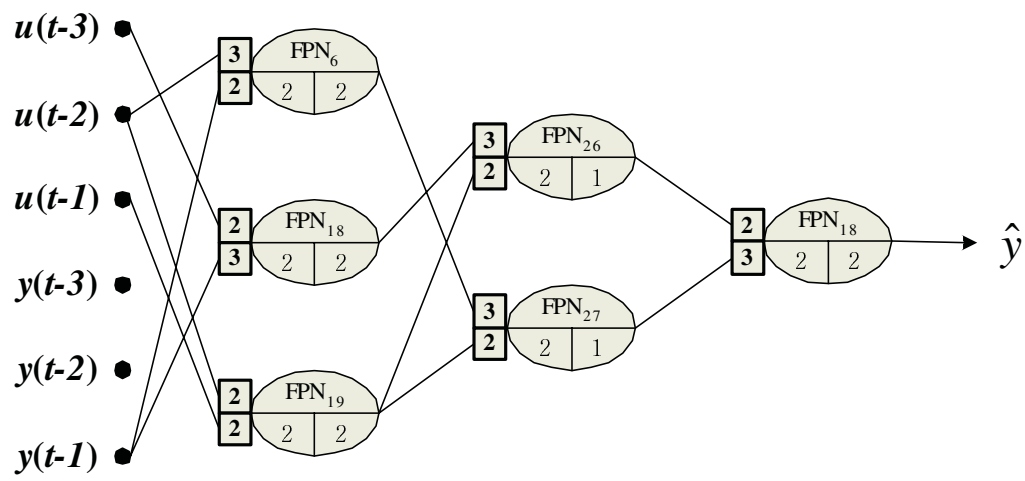

Fig. 2. Genetically dynamic optimization based FPNN (gdFPNN) architecture
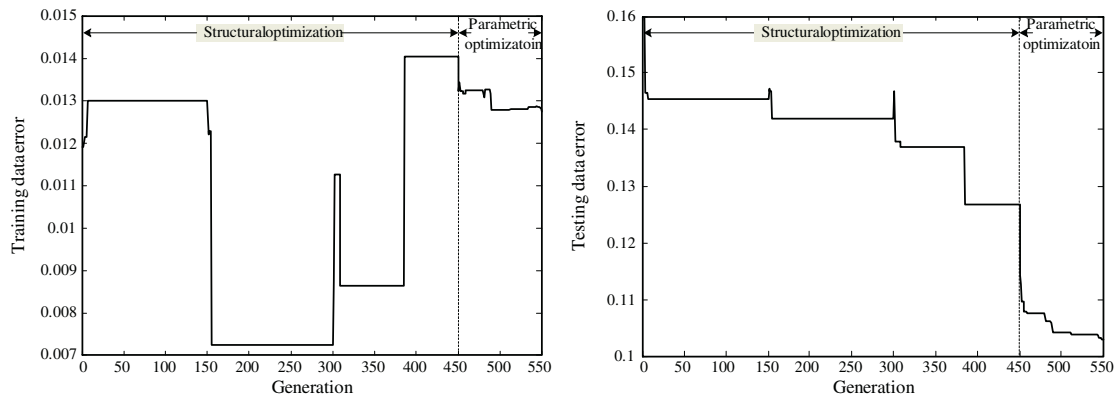

Fig. 3. The optimization process by the genetic algorithms 
Fig. 3 illustrates the optimization process by visualizing the values of the performance index obtained in successive generations of GA.

Table 2 gives a comparative summary of the network with other models.

Table 2. Comparative analysis of the performance of the network; considered are models reported in the literature

\begin{tabular}{|c|c|c|c|c|c|c|}
\hline \multicolumn{4}{|c|}{ Model } & PI & $\mathrm{PI}_{\mathrm{s}}$ & $\mathrm{EPI}_{s}$ \\
\hline \multicolumn{4}{|c|}{ Box and Jenkin's model [10] } & 0.710 & & \\
\hline \multicolumn{4}{|c|}{ Tong's model [11] } & 0.469 & & \\
\hline \multicolumn{4}{|c|}{ Sugeno and Yasukawa's model [12] } & 0.190 & & \\
\hline \multicolumn{4}{|c|}{$\mathrm{Xu}$ and Zailu's model [13] } & 0.328 & & \\
\hline \multicolumn{4}{|c|}{ Oh and Pedrycz's model [14] } & 0.123 & 0.020 & 0.271 \\
\hline \multicolumn{4}{|c|}{ Lin and Cunningham's model [15] } & & 0.071 & 0.261 \\
\hline \multirow{2}{*}{ FPNN [16] } & \multicolumn{3}{|c|}{ CASE I } & & 0.016 & 0.116 \\
\hline & & E II & & & 0.012 & 0.125 \\
\hline \multirow{2}{*}{ gFPNN [17] } & Triangular MF & & $\operatorname{Max}=4$ & & 0.018 & 0.122 \\
\hline & Gaussian-like MF & $3^{\text {rd }}$ & $\operatorname{Max}=4$ & & 0.020 & 0.104 \\
\hline \multirow{2}{*}{$\begin{array}{l}\text { Proposed } \\
\text { gdFPNN }\end{array}$} & Triangular MF & layer & $\operatorname{Max}=2$ & & 0.011 & 0.105 \\
\hline & Gaussian-like MF & & $\operatorname{Max}=3$ & & 0.007 & 0.106 \\
\hline
\end{tabular}

\section{Concluding Remarks}

In this study, the design procedure of genetically dynamic optimization based Fuzzy Polynomial Neural Networks (gdFPNN) along with their architectural considerations has been investigated. In contrast to the conventional FPNN structures and their learning, the proposed model comes with a diversity of local characteristics of FPNs that are extremely useful when coping with various nonlinear characteristics of the system under consideration. The design methodology comes as a structural and parametrical optimization being viewed as two fundamental phases of the design process. The comprehensive experimental studies involving well-known datasets quantify a superb performance of the network in comparison to the existing fuzzy and neuro-fuzzy models. Most importantly, through the proposed framework of genetic optimization we can efficiently search for the optimal network architecture (structurally and parametrically optimized network) and this becomes crucial in improving the performance of the resulting model.

Acknowledgement. This work was supported by Korea Research Foundation Grant (KRF-2004-002-D00257)

\section{References}

1. Cherkassky, V., Gehring, D., Mulier, F.: Comparison of adaptive methods for function estimation from samples. IEEE Trans. Neural Networks. 7 (1996) 969-984

2. Dickerson, J. A., Kosko, B.: Fuzzy function approximation with ellipsoidal rules. IEEE Trans. Syst., Man, Cybernetics. Part B. 26 (1996) 542-560 
3. Sommer, V., Tobias, P., Kohl, D., Sundgren, H., Lundstrom, L.: Neural networks and abductive networks for chemical sensor signals: A case comparison. Sensors and Actuators B. 28 (1995) 217-222

4. Kleinsteuber, S., Sepehri, N.: A polynomial network modeling approach to a class of large-scale hydraulic systems. Computers Elect. Eng. 22 (1996) 151-168

5. Cordon, O., et al.: Ten years of genetic fuzzy systems: current framework and new trends. Fuzzy Sets and Systems. 2003(in press)

6. Oh, S.K., Pedrycz, W.: Self-organizing Polynomial Neural Networks Based on PNs or FPNs : Analysis and Design. Fuzzy Sets and Systems. 142(2) (2003) 163-198

7. Oh, S.K., Pedrycz, W.: Fuzzy Polynomial Neuron-Based Self-Organizing Neural Networks. Int. J. of General Systems. 32 (2003) 237-250

8. Michalewicz, Z.: Genetic Algorithms + Data Structures = Evolution Programs. 3rd edn. Springer-Verlag, Berlin Heidelberg New York. (1996)

9. Jong, D., K. A.: Are Genetic Algorithms Function Optimizers?. Parallel Problem Solving from Nature 2, Manner, R. and Manderick, B. eds., North-Holland, Amsterdam. (1992)

10. Box, D.E., Jenkins, G.M.: Time Series Analysis, Forcasting and Control, California, Holden Day. (1976)

11. Tong, R.M.: The evaluation of fuzzy models derived from experimental data. Fuzzy Sets and Systems. 13 (1980) 1-12

12. Sugeno, M., Yasukawa, T.: A Fuzzy-Logic-Based Approach to Qualitative Modeling. IEEE Trans. Fuzzy Systems. 1 (1993) 7-31

13. $\mathrm{Xu}, \mathrm{C} . \mathrm{W} ., \mathrm{Xi}$, T.G., Zhang, Z.J.: A clustering algorithm for fuzzy model identification. Fuzzy Sets and Systems. 98 (1998) 319-329

14. Oh, S.K., Pedrycz, W.: Identification of Fuzzy Systems by means of an Auto-Tuning Algorithm and Its Application to Nonlinear Systems. Fuzzy Sets and Systems. 115 (2000) 205-230

15. Lin, Y., Cunningham III, G. A.: A new approach to fuzzy-neural modeling. IEEE Trans. Fuzzy Systems. 3 (1995) 190-197

16. Park, H.S., Oh, S.K., Yoon, Y.W.: A New Modeling Approach to Fuzzy-Neural Networks Architecture. Journal of Control, Automation and Systems Engineering. 7 (2001) 664674(in Koreans)

17. Oh, S.K., Pedrycz, W., Park, H.S.: Genetically Optimized Fuzzy Polynomial Neural Networks. IEEE Trans. Fuzzy Systems. (2004) (submitted)

18. Park, B.J., Lee, D.Y., Oh, S.K.: Rule-based Fuzzy Polynomial Neural Networks in Modeling Software Process Data. International journal of Control, Automations, and Systems. 1(3) (2003) 321-331 